\title{
The Association between Insulin Resistance and Cardiovascular Disease Risk: A Community-Based Cross-Sectional Study among Taiwanese People Aged over 50 Years
}

\author{
Mei-Chun Lu ${ }^{1,2,3}$, Wei-Ching Fang ${ }^{1}$, Wen-Cheng $\mathrm{Li}^{1,3,4}$, Wei-Chung Yeh ${ }^{1}$, Ying-Hua Shieh ${ }^{5}$ \\ and Jau-Yuan Chen $1,3, *$ (D) \\ 1 Department of Family Medicine, Chang-Gung Memorial Hospital, Linkou Branch, \\ Taoyuan City 33305, Taiwan; b101092026@tmu.edu.tw (M.-C.L.); winds75526@gmail.com (W.-C.F.); \\ wcl20130714@gmail.com (W.-C.L.); sendoh777777@gmail.com (W.-C.Y.) \\ 2 Department of Family Medicine, Chang Gung Memorial Hospital, Taoyuan Branch, \\ Taoyuan City 33378, Taiwan \\ 3 College of Medicine, Chang Gung University, Taoyuan City 33302, Taiwan \\ 4 Department of Health Management, Xiamen Chang-Gung Hospital, Xiamen 361000, China \\ 5 Department of Family Medicine, New Taipei Municipal TuCheng Hospital, New Taipei City 23652, Taiwan; \\ tony470120@gmail.com \\ * Correspondence: welins@cgmh.org.tw; Tel.: +886-975362672; Fax: +886-3-3287715
}

Received: 22 August 2020; Accepted: 29 September 2020; Published: 1 October 2020

\begin{abstract}
Background and Aims: Previous studies have implied that insulin resistance (IR) could represent a major underlying abnormality leading to cardiovascular disease (CVD). The aim of this study was to evaluate the relationships between IR (estimated by the homeostasis model assessment of IR (HOMA-IR) index) and CVD risk among middle-aged and elderly Taiwanese individuals. Methods: In this cross-sectional, community-based study, a total of 320 participants were interviewed to collect demographical parameters and blood samples. The recruited participants were divided into tertiles according to their levels of HOMA-IR. The Framingham risk score (FRS) was calculated according to the 2008 general CVD risk model from the Framingham Heart Study. Results: The HOMA-IR index was significantly correlated with the FRS, with a Pearson's coefficient of 0.22 . In the multiple logistic regression model, a higher HOMA-IR level was significantly associated with a high FRS (FRS $\geq 20 \%$ ) (highest tertile vs. lowest tertile of HOMA-IR, crude OR $=3.69 ; 95 \% \mathrm{CI}=1.79-7.62$ ), even after adjusting for smoking, fasting plasma glucose (FPG), and systolic blood pressure (SBP) (highest tertile vs. lowest tertile of HOMA-IR, adjusted $\mathrm{OR}=11.51 ; 95 \% \mathrm{CI}=2.55-51.94$ ). The area under the receiver operating characteristic curve for the HOMA-IR index as the predictor of high FRS was 0.627 , and the optimal HOMA-IR cutoff value was 1.215 (sensitivity $=83.6 \%$, specificity $=42.9 \%$ ). Conclusions: We considered that HOMA-IR is an independent factor but that it cannot be used solely for evaluating the CVD risk due to the low AUC value. Further prospective cohort studies are warranted to better assess the relationship between CVD risk and insulin resistance.
\end{abstract}

Keywords: insulin resistance; HOMA-IR; prediction; CVD; Framingham risk score

\section{Introduction}

Insulin resistance (IR) is thought to be the key mechanism of metabolic syndrome, a cluster of cardiovascular risk factors [1-6]. Previous studies have shown that hyperinsulinemia was an independent predictor of incident myocardial ischemic events in a non-diabetic population [7]. IR is also an independent risk factor for various chronic diseases, such as hypertension [8], hepatic 
steatosis and steatohepatitis [9], chronic kidney disease [10], and even lung cancer [11] and Alzheimer's disease [12].

Overnutrition, obesity, and specific dietary components can trigger IR [9,13]. Excess daily calories, fructose, and saturated fatty acid intake; a lack of n-3 polyunsaturated fatty acids and antioxidants; as well as intestinal microbiome imbalance can increase the oxidative stress in the liver and lead to IR and non-alcoholic hepatic steatosis and steatohepatitis [9].

The hyperinsulinemic-euglycemic clamp technique is the gold standard for evaluating insulin resistance, but it is impractical in clinical practice. Thus, surrogate markers such as the homeostasis model assessment of IR (HOMA-IR) have been developed to assess IR [14]. In Taiwan, the 2008 version of Framingham risk score (FRS) is frequently used by clinicians to estimate a person's risk of cardiovascular disease (CVD) in the next 10 years. Additionally, previous studies have shown that the FRS is applicable in Asian populations [15]. However, only limited studies have discussed the association of HOMA-IR with FRS [16,17]. For example, obese children with an increased HOMA-IR have a higher CVD risk [17].

Therefore, the aim of the current study was to investigate the associations between the HOMA-IR index and FRS and evaluate the ability of HOMA-IR to identify community residents with a high FRS (FRS $\geq 20 \%$ ) in middle-aged and elderly Taiwanese individuals.

\section{Materials and Methods}

\subsection{Participants}

This was a cross-sectional study. The participants were recruited through a community health promotion project in Linkou Chang Gung Memorial Hospital in Taiwan from February 2014 to August 2014. The project was approved by the Institutional Review Board (102-2304B) of Linkou Chang Gung Memorial Hospital, and informed consent was signed by all the participants before enrollment.

A total of 400 participants aged over 50 years living in Guishan district (a rural district in northeastern Taiwan) were recruited. General information, including medical history and lifestyle, was obtained by appropriately trained interviewers. Participants with a history of CVD $(n=19)$, aged 75 years and above $(n=56)$, or with incomplete data $(n=3)$ were excluded. Extreme outliers of HOMA-IR $(n=2)$ were excluded. Finally, 320 participants were included in this study.

\subsection{Anthropometric}

Detailed anthropometric measurements, such as height, weight, waist circumference (WC), and blood pressure, were conducted by trained research assistants or nurses under the supervision of a medical doctor. The measurement of heights was carried out with the subjects standing with their feet together. Body mass index (BMI) was calculated by dividing weight by the square of the height $\left(\mathrm{kg} / \mathrm{m}^{2}\right)$. Blood pressure (BP) was measured using an automated sphygmomanometer after the participants were relaxed and seated for more than $10 \mathrm{~min}$. The WC was measured halfway between the iliac crest and the lowest rib at the end of a relaxed expiration. The height meter and weight scale were calibrated once per day.

\subsection{Laboratory Examinations}

Participants were requested to fast for at least $12 \mathrm{~h}$ and avoid a high-fat diet or alcohol consumption before blood sampling. Blood samples were stored in a refrigerator at $4{ }^{\circ} \mathrm{C}$ and then sent to the hospital laboratory. Clinical biochemistry tests were performed in a hospital laboratory accredited by the College of American Pathologists. The fasting plasma glucose (FPG); fasting insulin; alanine aminotransferase (ALT); creatinine; uric acid; and fasting lipid profiles, such as triglyceride (TG), high-density lipoprotein cholesterol (HDL-C), and low-density lipoprotein cholesterol (LDL-C), were measured. 


\subsection{Assessment of Insulin Resistance}

We assessed insulin resistance by HOMA-IR [14], which is expressed as:

$$
\text { HOMA }-\mathrm{IR}=\frac{\text { fasting glucose }(\text { in } \mathrm{mmol} / \mathrm{L}) \times \text { fasting insulin }(\mathrm{in} \mathrm{mU} / \mathrm{ml})}{22.5}
$$

Subjects were divided into tertiles based on the HOMA-IR index:

Low HOMA-IR group: HOMA-IR $\leq 1.15$;

Middle HOMA-IR group: $1.15<$ HOMA-IR $\leq 1.93$;

High HOMA-IR group: HOMA-IR $>1.93$.

\subsection{Cardiovascular Risk Assessments}

We used the 2008 Framingham risk score (FRS) assessments to determine the risk of CVD [18]. The risk factors and the equations used to calculate the FRS are listed below:

Women

$$
\begin{aligned}
& \text { Risk Factors }=(\ln (\text { Age }) \times 2.32888)+(\ln (\text { Total cholesterol }) \times 1.20904) \\
&-(\ln (\text { HDL Cholesterol }) \times 0.70833) \\
&+(\ln (\text { Systolic blood pressure }) \times \text { blood pressure medication factor }) \\
&+ \text { Cigarette smoker factor }+ \text { Diabetes present factor }-26.1931 \\
& \text { FRS }=100 \times\left((1-0.95012)^{\mathrm{r}}\right) \\
& \mathrm{r}=\mathrm{e}^{(\text {Risk factors })}
\end{aligned}
$$

Men

$$
\begin{aligned}
& \text { Risk Factors }=(\ln (\text { Age }) \times 3.06117)+(\ln (\text { Total cholesterol }) \times 1.12370) \\
&-(\ln (\text { HDL Cholesterol }) \times 0.93263) \\
&+(\ln (\text { Systolic blood pressure }) \times \text { blood pressure medication factor }) \\
&+ \text { Cigarette smoker factor }+ \text { Diabetes present factor }-23.9802 \\
& \text { FRS }=100 \times\left((1-0.88936)^{\mathrm{r}}\right) \\
& \mathrm{r}=\mathrm{e}^{(\text {Risk factors })}
\end{aligned}
$$

A high FRS was defined as an FRS $\geq 20 \%$.

\subsection{Statistical Analysis}

Demographics and clinical characteristics are presented as means with standard deviations and counts with percentages for continuous and discrete variables, respectively. Descriptive statistics were presented, and differences between groups with different HOMA-IR levels were compared using one-way ANOVA for continuous data and the chi-square test for categorical data. The correlation of cardiovascular disease risk factors and HOMA-IR levels was assessed using Pearson's analysis. Multiple logistic regression analysis was used to adjust for covariates. A receiver operating characteristic (ROC) curve was constructed to assess the performance of the HOMA-IR to predict a high FRS. Youden's index, which maximizes the sum of sensitivity and specificity (max (sensitivity+ specificity-1)), was used to determine the optimal cutoff point, and the corresponding sensitivity, specificity, and area under the receiver operating characteristic curve (AUC) were calculated. All the statistical tests were regarded as statistically significant, with a $p$-value less than 0.05 (two-sided). Data were analyzed using the Statistical Package for the Social Sciences software, version 22.0 (SPSS Inc., Chicago, IL, USA). 


\section{Results}

\subsection{Baseline Characteristics of the Patient Population}

The general characteristics of the participants according to the tertile of the HOMA-IR level are summarized in Table 1. Overall, the mean age was $61.88 \pm 6.21$ years, and the mean FRS was $14.31 \pm$ $11.47 \%$. There were no significant differences between the three groups concerning age, creatinine, smoking, alcohol drinking, and regular exercise. However, the mean FRS was significantly higher in the groups with higher HOMA-IR levels. The mean FRS were $10.95 \pm 7.85 \%$ (low HOMA-IR group), $13.94 \pm 10.21 \%$ (middle HOMA-IR group), and $18.04 \pm 14.33 \%$ (high HOMA-IR group), respectively. Other parameters with significant differences between the three groups with $p$-values lower than 0.05 were SBP, BMI, WC, ALT, FPG, HDL-C, TG, LDL-C, and uric acid. Figure 1 shows that the prevalence of high FRS was higher among the high HOMA-IR tertile than among the two lower tertiles ( $p$-value for the Cochran-Armitage trend test of <0.001).

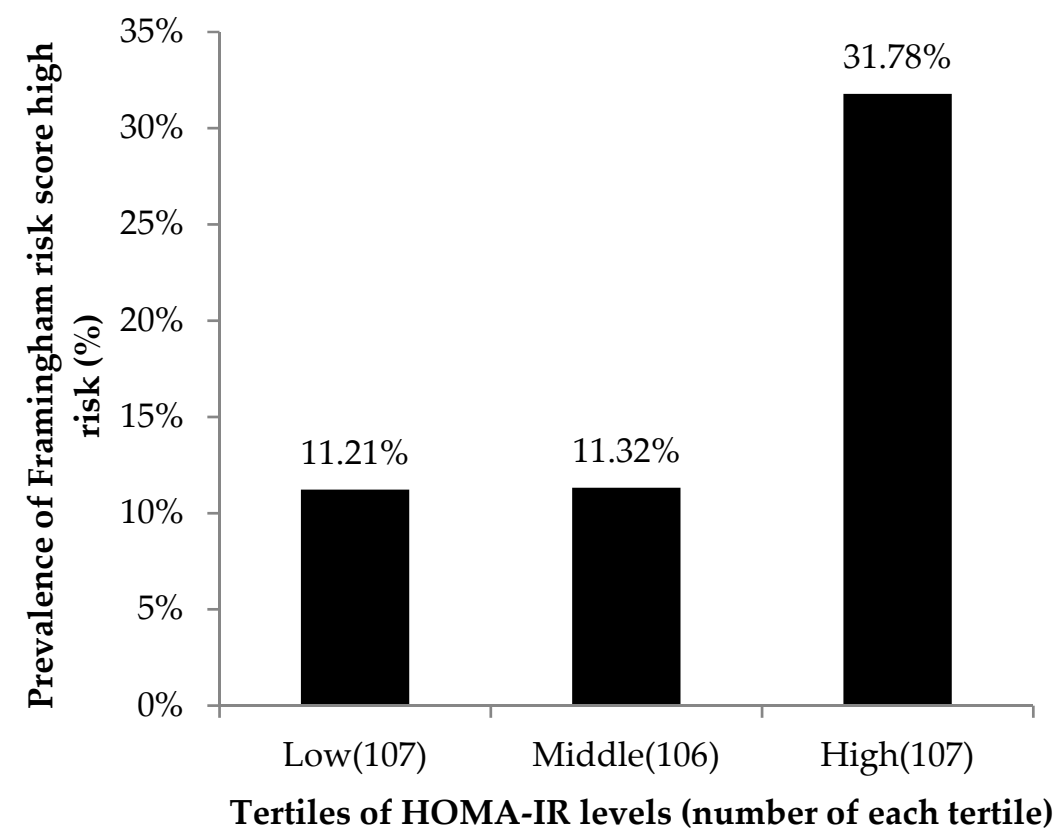

Figure 1. Prevalence of high FRS (FRS $\geq 20 \%$ ) according to the tertiles of the HOMA-IR index levels. The prevalence of high FRS was higher among the high HOMA-IR tertile than among the two lower tertiles. 
Table 1. General characteristics of the study population according to the tertiles of the HOMA-IR levels.

\begin{tabular}{|c|c|c|c|c|c|c|c|c|c|}
\hline \multirow{3}{*}{$\begin{array}{l}\text { Variables } \\
\text { Age (year) }\end{array}$} & \multicolumn{9}{|c|}{ HOMA-IR Levels } \\
\hline & \multicolumn{2}{|c|}{$\begin{array}{c}\text { Total } \\
(n=320)\end{array}$} & \multicolumn{2}{|c|}{$\begin{array}{c}\text { Low } \\
(\text { HOMA-IR } \leq 1.15) \\
(n=107)\end{array}$} & \multicolumn{2}{|c|}{$\begin{array}{c}\text { Middle } \\
(1.15<\text { HOMA-IR } \leq 1.93) \\
(n=106)\end{array}$} & \multicolumn{2}{|c|}{$\begin{array}{c}\text { High } \\
(\text { HOMA-IR > 1.93) } \\
(n=107)\end{array}$} & \multirow{2}{*}{$\begin{array}{c}p \text {-value } \\
0.38\end{array}$} \\
\hline & 61.88 & \pm 6.21 & 61.38 & \pm 6.13 & 61.72 & \pm 6.54 & 62.53 & \pm 5.93 & \\
\hline $\mathrm{SBP}(\mathrm{mmHg})$ & 128.76 & \pm 16.09 & 124.52 & \pm 16.77 & 129.20 & \pm 14.41 & 132.56 & \pm 16.11 & 0.001 \\
\hline $\mathrm{DBP}(\mathrm{mmHg})$ & 78.12 & \pm 10.80 & 76.80 & \pm 11.56 & 78.01 & \pm 9.97 & 79.55 & \pm 10.72 & 0.176 \\
\hline BMI $\left(\mathrm{kg} / \mathrm{m}^{2}\right)$ & 24.61 & \pm 3.52 & 22.95 & \pm 2.99 & 24.55 & \pm 2.82 & 26.32 & \pm 3.84 & $<0.001$ \\
\hline Waist circumference $(\mathrm{cm})$ & 84.54 & \pm 9.37 & 80.31 & \pm 7.52 & 83.62 & \pm 8.04 & 89.68 & \pm 9.89 & $<0.001$ \\
\hline $\operatorname{ALT}(\mathrm{U} / \mathrm{L})$ & 22.89 & \pm 12.89 & 19.61 & \pm 7.49 & 22.18 & \pm 11.71 & 26.89 & \pm 16.75 & $<0.001$ \\
\hline Creatinine (mg/dL) & 0.72 & \pm 0.28 & 0.69 & \pm 0.16 & 0.73 & \pm 0.32 & 0.74 & \pm 0.34 & 0.37 \\
\hline eGFR $\left(\mathrm{ml} / \mathrm{min} / 1.73 \mathrm{~m}^{2}\right)$ & 117.43 & \pm 30.65 & 121.03 & \pm 28.94 & 116.23 & \pm 31.53 & 115.01 & \pm 31.37 & 0.32 \\
\hline $\mathrm{FPG}(\mathrm{mg} / \mathrm{dL})$ & 95.33 & \pm 21.96 & 85.065 & \pm 8.44 & 92.113 & \pm 12.37 & 108.77 & \pm 30.49 & $<0.001$ \\
\hline HDL-C (mg/dL) & 54.96 & \pm 13.83 & 61.037 & \pm 14.67 & 54.472 & \pm 12.36 & 49.36 & \pm 11.84 & $<0.001$ \\
\hline HOMA-IR index & 1.85 & \pm 1.36 & 0.80 & \pm 0.23 & 1.51 & \pm 0.23 & 3.24 & \pm 1.52 & $<0.001$ \\
\hline LDL-C (mg/dL) & 120.20 & \pm 31.85 & 121.77 & \pm 34.23 & 124.78 & \pm 30.51 & 114.10 & \pm 29.96 & 0.04 \\
\hline Triglyceride (mg/dL) & 123.37 & \pm 65.14 & 93.97 & \pm 41.90 & 119.44 & \pm 54.73 & 156.66 & \pm 77.71 & $<0.001$ \\
\hline Uric Acid (mg/dL) & 5.71 & \pm 1.41 & 5.39 & \pm 1.30 & 5.71 & \pm 1.41 & 6.03 & \pm 1.45 & 0.003 \\
\hline Framingham risk score $(\%)$ & 14.31 & \pm 11.47 & 10.95 & \pm 7.85 & 13.94 & \pm 10.21 & 18.04 & \pm 14.33 & $<0.001$ \\
\hline Current smoking, $n(\%)$ & 35 & $(10.9)$ & 13 & $(12.1)$ & 10 & $(9.4)$ & 12 & $(11.2)$ & 0.81 \\
\hline $\begin{array}{c}\text { Alcohol drinking } \geq 2 \\
\text { times/week, } n(\%)\end{array}$ & 62 & $(19.4)$ & 26 & $(24.3)$ & 21 & (19.8) & 15 & $(14.0)$ & 0.16 \\
\hline Regular exercise, $n(\%)$ & 259 & $(80.9)$ & 92 & $(86.0)$ & 87 & $(82.1)$ & 80 & $(74.8)$ & 0.11 \\
\hline $\mathrm{HTN}, n(\%)$ & 152 & $(47.5)$ & 40 & $(37.4)$ & 42 & $(39.6)$ & 70 & $(65.4)$ & $<0.001$ \\
\hline $\mathrm{DM}, n(\%)$ & 54 & $(16.9)$ & 2 & $(1.9)$ & 13 & $(12.3)$ & 39 & $(36.4)$ & $<0.001$ \\
\hline Hyperlipidemia, $n(\%)$ & 211 & $(65.9)$ & 60 & $(56.1)$ & 70 & $(66.0)$ & 81 & $(75.7)$ & 0.01 \\
\hline
\end{tabular}

Notes: Clinical characteristics are expressed as the mean $\pm \mathrm{SD}$ for continuous variables and $n$ (\%) for categorical variables. $p$-values were derived from a one-way analysis of variance (one-way ANOVA) for continuous variables and chi-square tests for categorical variables. Abbreviations: BMI, body mass index; SBP, systolic blood pressure; DBP, diastolic blood pressure; ALT, alanine aminotransferase; FPG, fasting plasma glucose; HDL-C, high-density lipoprotein cholesterol; LDL-C, low-density lipoprotein cholesterol; TG, triglyceride; HTN, hypertension. 


\subsection{Correlation Analysis}

The correlations of the HOMA-IR levels with the CVD risk factors are shown in Table 2. The factors with significant positive correlations with IR were SBP, BMI, FRS, WC, FPG, TG, and uric acid, while HDL-C and LDL-C showed significant negative correlations with IR. Among them, BMI, WC, FPG, and TG showed a stronger correlation with IR than the correlation of the FRS with IR. Considering that the negative correlation of LDL-C was very weak (Pearson's coefficient -0.11), we did not involve LDL-C in the subsequent multiple logistic regression analysis. Figure 2 demonstrates the correlation of FRS and IR with a Pearson's coefficient of 0.22.

Table 2. Pearson correlation coefficient of the HOMA-IR levels with the cardiovascular disease risk factors.

\begin{tabular}{ccc}
\hline & \multicolumn{2}{c}{ HOMA-IR Index $(\boldsymbol{n}=\mathbf{3 2 0})$} \\
\hline Variables & Pearson's coefficient & $p$-value \\
Age (year) & 0.02 & 0.68 \\
SBP $(\mathrm{mmHg})$ & 0.16 & 0.005 \\
DBP $(\mathrm{mmHg})$ & 0.09 & 0.126 \\
BMI $\left(\mathrm{kg} / \mathrm{m}^{2}\right)$ & 0.46 & $<0.001$ \\
Framingham risk score $(\%)$ & 0.22 & $<0.001$ \\
Waist circumference $(\mathrm{cm})$ & 0.45 & $<0.001$ \\
FPG $(\mathrm{mg} / \mathrm{dL})$ & 0.58 & $<0.001$ \\
HDL-C $(\mathrm{mg} / \mathrm{dL})$ & -0.32 & $<0.001$ \\
TG $(\mathrm{mg} / \mathrm{dL})$ & 0.34 & $<0.001$ \\
LDL-C $(\mathrm{mg} / \mathrm{dL})$ & -0.11 & 0.04 \\
Uric Acid $(\mathrm{mg} / \mathrm{dL})$ & 0.16 & 0.003 \\
\hline
\end{tabular}

Abbreviations: SBP, systolic blood pressure; DBP, diastolic blood pressure; BMI, body mass index; FPG, fasting plasma glucose; HDL-C, high-density lipoprotein cholesterol; LDL-C, low-density lipoprotein cholesterol; TG, triglyceride.

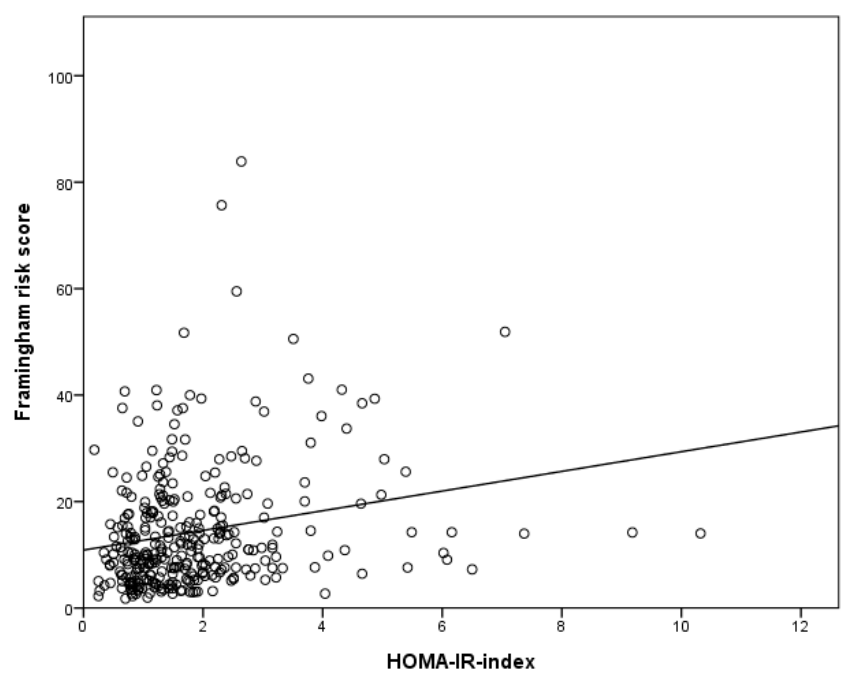

Figure 2. Correlation coefficients between the HOMA-IR levels and the Framingham risk score.

\subsection{Association between the Tertiles of HOMA-IR Levels and the Framingham Risk Factors}

Table 3 shows the results of the multiple logistic regression analyses in comparing the risk of high FRS between subjects with different HOMA-IR levels (with the low HOMA-IR level group as the reference group). In Model 1 (unadjusted), the odds ratios (ORs) for the middle and high HOMA-IR groups were 2.71 (95\% CI: 1.29-5.69, $p$-value 0.009 ) and 3.69 (95\% CI: 1.79-7.62, $p$-value $<0.001$ ), respectively. In Model 2, the ORs (95\% CI) were determined after further adjustment for gender. In Model 3, the ORs (95\% CI) were determined after further adjustment for gender, age, and BMI. In Model 4, the HOMA-IR level remained significantly correlated with high FRS after adjustment for 
covariates, including age, gender, BMI, smoking status, SBP, and FPG. The adjusted ORs for the middle HOMA-IR and high HOMA-IR groups were 11.31 (95\% CI: 2.94-43.52, $p$-value < 0.001) and 11.51 (95\% CI: $2.55-51.94, p$-value $=0.001$, , respectively.

\subsection{ROC Analysis}

The AUC for the HOMA-IR index as a predictor of high FRS was 0.627 , as shown in Table 4 and Figure 3. According to Youden's index, the optimal cutoff point for the HOMA-IR index for predicting a high FRS was 1.215 , with a sensitivity and a specificity of $83.6 \%$ and $42.9 \%$, respectively.

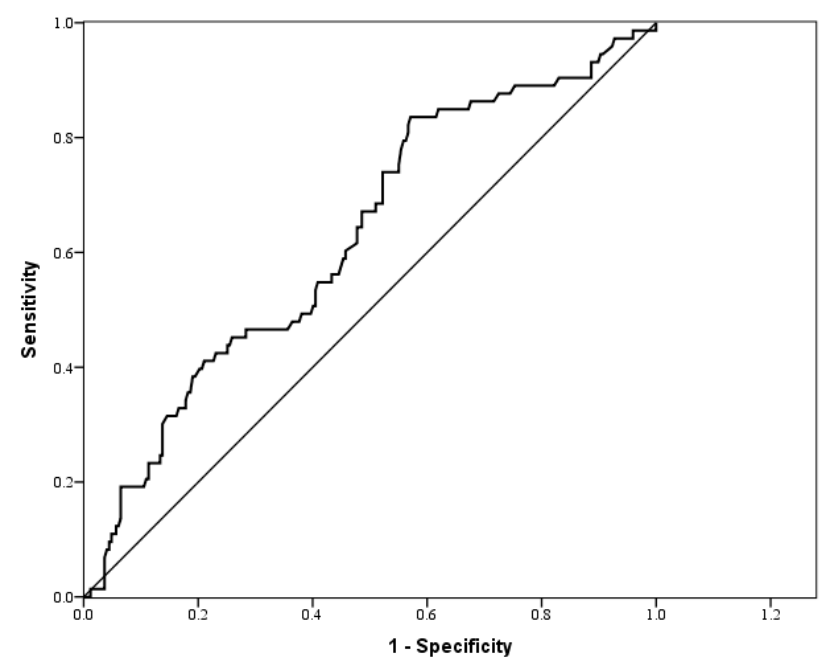

Figure 3. ROC curve for the HOMA-IR index as a predictor of the Framingham risk score. 
Table 3. Association between the tertiles of the HOMA-IR levels and a high FRS (FRS $\geq 20 \%$ ).

\begin{tabular}{|c|c|c|c|c|c|c|c|c|c|c|c|c|}
\hline \multirow[b]{2}{*}{ Variables } & \multicolumn{3}{|c|}{ Model 1} & \multicolumn{3}{|c|}{ Model 2} & \multicolumn{3}{|c|}{ Model 3} & \multicolumn{3}{|c|}{ Model 4} \\
\hline & OR & $(95 \% \mathrm{CI})$ & $p$-value & OR & $(95 \% \mathrm{CI})$ & $p$-value & OR & $(95 \% \mathrm{CI})$ & $p$-value & OR & $(95 \%$ CI $)$ & $p$-value \\
\hline Low & 1.00 & & & 1.00 & & & 1.00 & & & 1.00 & & \\
\hline Middle & 2.71 & $(1.29-5.69)$ & 0.009 & 5.16 & $(2.12-12.57)$ & $<0.001$ & 5.66 & $(2.17-14.78)$ & $<0.001$ & 11.31 & $(2.94-43.52)$ & $<0.001$ \\
\hline High & 3.69 & $(1.79-7.62)$ & $<0.001$ & 9.67 & (3.83-24.37) & $<0.001$ & 9.01 & $(3.14-25.81)$ & $<0.001$ & 11.51 & $(2.55-51.94)$ & 0.001 \\
\hline$p$-value for trend & & & $<0.001$ & & & $<0.001$ & & & $<0.001$ & & & 0.002 \\
\hline
\end{tabular}

Model 1: unadjusted; Model 2: adjusted for sex; Model 3: adjusted for factors in model 2 plus age and BMI; Model 4: adjusted for factors in model 3 plus smoking, FPG, SBP. Abbreviations: BMI, body mass index; FPG, fasting plasma glucose; SBP, systolic blood pressure; $\mathrm{CI}$, confidence interval.

Table 4. The areas under the ROC curve (AUC), sensitivity, and specificity of the optimized cut-off points for the HOMA-IR index in predicting high FRS (FRS $\geq 20 \%$ ).

\begin{tabular}{cccccc}
\hline Variables & AUC (95\% CI) & $p$ Value & Cut-off point & Sensitivity & Specificity \\
\hline HOMA-IR index & 0.627 & 0.001 & 1.215 & 0.836 & 0.429 \\
\hline \multicolumn{7}{c}{ Abbreviations: ROC curve, receiver operating characteristic curve; CI, confidence interval. }
\end{tabular}

Abbreviations: ROC curve, receiver operating characteristic curve; $\mathrm{CI}$, confidence interval. 


\section{Discussion}

Our study objective was to investigate the associations between IR and future CVD risk. In this community-based cross-sectional study among Taiwanese people aged over 50 years, the level was positively associated with the FRS after adjusting for potential confounders. Several studies have reported a positive correlation between CVD risk and IR [19-22], which is consistent with our findings.

HOMA-IR is a frequently used tool to estimate IR. However, there are only limited studies focusing on the use of the HOMA-IR level for predicting high FRS. In our study, participants with high HOMA-IR levels had a higher prevalence of high FRS compared with those with lower HOMA-IR levels (Figure 1). After adjusting for other risk factors, including sex, age, BMI, current smoking status, FPG, and SBP through multiple logistic regression models, the HOMA-IR level was still significantly associated with high FRS. These results reinforce that HOMA-IR is an independent risk factor for high FRS. We then evaluated the ability of HOMA-IR to identify community residents with a high FRS. However, due to the low specificity and low AUC value, HOMA-IR cannot be used as the sole predictor of high FRS.

The recent studies have revealed that there are several mechanisms to induce IR, such as genetic defects, overnutrition, obesity, and inflammation $[13,23]$. In overnutrition status, the excess intra-cellular free fatty acids will lead to the activation of PKC and NFKB, then induce serine 307 phosphorylation for IRS-1, which inhibits the insulin signal pathway and eventually results in IR in the skeleton muscle and hepatocytes. The PKC activation and increased free fatty acid flux to the liver results in hepatosteatosis $[13,24,25]$.

Excess free fatty acids in cardiomyocytes and endothelial cells also cause serine 307 phosphorylation for IRS-1, then inhibit the insulin signaling pathway [23,26]. An inhibited insulin signaling pathway results in increased free fatty acid oxidation and decreased glucose oxidation in cardiomyocyte and causes cell death [23]. In the endothelial cells, IR causes an imbalance between the production of eNO and the secretion of endothelin-1, resulting in endothelial dysfunction [26].

The early detection of IR, as well as interventions in lifestyle and dietary components, could decrease the development and progression of IR and reverse further damage to the liver, heart, and endothelium. In Taiwan, free adult health exams offered by the Health Promotion Administration cover all the needed parameters for FRS calculation and fasting glucose, but not fasting insulin. Besides, clinicians do not routinely check HOMA-IR. For individuals with diabetes or obesity, it is easy to link their condition with insulin resistance. However, for high FRS individuals who are elderly, smokers, or have hypertension, it is harder for clinicians to think about IR. This study revealed that HOMA-IR is an independent risk factor for high FRS. Clinicians should be more alert for IR in high-FRS individuals, and offer lifestyle and dietary interventions earlier for better prognosis.

Lifestyle interventions and dietary interventions are reported to improve IR, the lipid profile, and liver function enzymes [9,13]. Lifestyle interventions include body weight loss of over 5\% of the initial weight, physical activity for $150 \mathrm{~min} /$ week or more, training exercises for four weeks, or a regular exercise program. Dietary interventions include daily calorie restriction by reducing $500 \mathrm{Kcal}$ from usual food consumption, a high-protein diet, fructose restriction, and choosing a Mediterranean diet. Supplementation with anti-oxidatives, amino acids, vitamins, n-3 polyunsaturated fatty acids, and prebiotics/probiotics may have potential benefits, but lack sufficient evidence [9].

This study still has some limitations. First, the study was cross-sectional; therefore, it is unable to explore the cause-effect relationship between FRS and IR. Second, we do not have a direct outcome measure of CVD. Third, we did not check Hba1c, a more reliable marker for identifying pre-diabetic and diabetic individuals. Finally, our sample size was relatively small. Further studies are warranted with unknown confounders.

\section{Conclusions}

In conclusion, a high FRS (FRS $\geq 20 \%$ ) was significantly associated with IR in the middle-aged and elderly population in Taiwan. HOMA-IR is an independent risk factor for high FRS, but cannot be 
used as the sole predictor due to its low specificity. Further prospective cohort studies are warranted to better assess the relationship between CVD risk and insulin resistance in middle-aged and elderly Taiwanese populations.

Author Contributions: Conceptualization, W.-C.L. and J.-Y.C.; methodology, J.-Y.C.; software, J.-Y.C.; validation, J.-Y.C.; formal analysis, J.-Y.C.; investigation, M.-C.L., W.-C.F., and J.-Y.C.; resources, Y.-H.S. and J.-Y.C.; data curation, M.-C.L. and J.-Y.C.; writing-original draft preparation, M.-C.L.; writing-review and editing, M.C.L.; visualization, W.-C.F. and J.-Y.C.; supervision, W.-C.L. and J.-Y.C.; project administration, W.-C.Y. and J.-Y.C.; funding acquisition, J.-Y.C. All authors have read and agreed to the published version of the manuscript.

Funding: This study was supported by Chang Gung Memorial Hospital (grants CORPG3C0171, CORPG3C0172, CZRPG3C0053, CORPG3G0021, CORPG3G0022, and CORPG3G0023).

Conflicts of Interest: The authors declare no conflict of interest.

\section{Abbreviations}

$\begin{array}{ll}\text { HOMA-IR } & \text { homeostasis model assessment of insulin resistance } \\ \text { IR } & \text { insulin resistance } \\ \text { CVD } & \text { cardiovascular disease } \\ \text { CKD } & \text { chronic kidney disease } \\ \text { WC } & \text { waist circumference } \\ \text { FRS } & \text { Framingham risk score } \\ \text { BMI } & \text { body mass index } \\ \text { BP } & \text { blood pressure } \\ \text { FPG } & \text { fasting plasma glucose } \\ \text { HDL-C } & \text { high-density lipoprotein cholesterol } \\ \text { LDL-C } & \text { low-density lipoprotein cholesterol } \\ \text { TG } & \text { triglyceride } \\ \text { ALT } & \text { alanine aminotransferase } \\ \text { eGFR } & \text { estimated glomerular filtration rate } \\ \text { ROC } & \text { receiver operating characteristic } \\ \text { AUC } & \text { area under the ROC curve } \\ \text { OR } & \text { odds ratio } \\ \text { aOR } & \text { adjusted odds ratio }\end{array}$

\section{References}

1. DeFronzo, R. Insulin resistance: a multifaceted syndrome responsible for NIDDM, obesity, hypertension, dyslipidaemia and atherosclerosis. Neth. J. Med. 1997, 50, 191-197. [CrossRef]

2. Beilby, J.P. Definition of Metabolic Syndrome: Report of the National Heart, Lung, and Blood Institute/American Heart Association Conference on Scientific Issues Related to Definition. Clin. Biochem. Rev. 2004, 25, 195-198.

3. Girman, C.J.; Rhodes, T.; Mercuri, M.; Pyörälä, K.; Kjekshus, J.; Pedersen, T.R.; A Beere, P.; Gotto, A.M.; Clearfield, M. The metabolic syndrome and risk of major coronary events in the Scandinavian Simvastatin Survival Study (4S) and the Air Force/Texas Coronary Atherosclerosis Prevention Study (AFCAPS/TexCAPS). Am. J. Cardiol. 2004, 93, 136-141. [CrossRef] [PubMed]

4. Lakka, H.-M.; Laaksonen, D.E.; Lakka, T.; Niskanen, L.K.; Kumpusalo, E.; Tuomilehto, J.; Salonen, J.T. The metabolic syndrome and total and cardiovascular disease mortality in middle-aged men. JAMA 2002, 288, 2709-2716. [CrossRef] [PubMed]

5. Eckel, R.; Alberti, K.; Grundy, S.M.; Zimmet, P. The metabolic syndrome. Lancet 2010, 375, 181-183. [CrossRef]

6. Reaven, G.M. Banting lecture 1988. Role of insulin resistance in human disease. Diabetes 1988, 37, $1595-1607$. [CrossRef]

7. Després, J.-P.; Lamarche, B.; Mauriège, P.; Cantin, B.; Dagenais, G.R.; Moorjani, S.; Lupien, P.-J. Hyperinsulinemia as an Independent Risk Factor for Ischemic Heart Disease. New Engl. J. Med. 1996, 334, 952-958. [CrossRef] 
8. Wang, F.; Han, L.; Hu, D. Fasting insulin, insulin resistance and risk of hypertension in the general population: A meta-analysis. Clin. Chim. Acta 2017, 464, 57-63. [CrossRef]

9. Hernandez-Rodas, M.C.; Valenzuela, R.; A Videla, L. Relevant Aspects of Nutritional and Dietary Interventions in Non-Alcoholic Fatty Liver Disease. Int. J. Mol. Sci. 2015, 16, 25168-25198. [CrossRef]

10. E Kosmas, C.; Silverio, D.; Tsomidou, C.; Salcedo, M.D.; Montan, P.D.; Guzman, E. The Impact of Insulin Resistance and Chronic Kidney Disease on Inflammation and Cardiovascular Disease. Clin. Med. Insights: Endocrinol. Diabetes 2018, 11. [CrossRef]

11. Petridou, E.T.; Sergentanis, T.; Antonopoulos, C.N.; Dessypris, N.; Matsoukis, I.L.; Aronis, K.N.; Efremidis, A.; Syrigos, C.; Mantzoros, C.S. Insulin resistance: an independent risk factor for lung cancer? Metab. 2011, 60, 1100-1106. [CrossRef] [PubMed]

12. Ma, L.; Wang, J.; Li, Y. Insulin resistance and cognitive dysfunction. Clin. Chim. Acta 2015, 444, 18-23. [CrossRef] [PubMed]

13. Valenzuela, R.; Videla, L.A. The importance of the long-chain polyunsaturated fatty acid n-6/n-3 ratio in development of non-alcoholic fatty liver associated with obesity. Food Funct. 2011, 2, 644-648. [CrossRef]

14. Matthews, D.R.; Hosker, J.P.; Rudenski, A.S.; Naylor, B.A.; Treacher, D.F.; Turner, R.C. Homeostasis model assessment: insulin resistance and ?-cell function from fasting plasma glucose and insulin concentrations in man. Diabetol. 1985, 28, 412-419. [CrossRef] [PubMed]

15. Selvarajah, S.; Kaur, G.; Haniff, J.; Cheong, K.C.; Hiong, T.G.; Van Der Graaf, Y.; Bots, M.L. Comparison of the Framingham Risk Score, SCORE and WHO/ISH cardiovascular risk prediction models in an Asian population. Int. J. Cardiol. 2014, 176, 211-218. [CrossRef] [PubMed]

16. Li, X.; Wang, F.; Xu, H.; Qian, Y.; Zou, J.; Yang, M.; Zhu, H.; Yi, H.; Guan, J.; Yin, S. Interrelationships among common predictors of cardiovascular diseases in patients of OSA: A large-scale observational study. Nutr. Metab. Cardiovasc. Dis. 2020, 30, 23-32. [CrossRef]

17. Ferreira, A.P.; Oliveira, C.E.R.; De França, N.M. Metabolic syndrome and risk factors for cardiovascular disease in obese children: the relationship with insulin resistance (HOMA-IR). J. de Pediatr. 2007, 83, 21-26. [CrossRef]

18. D'Agostino, R.B.; Vasan, R.S.; Pencina, M.J.; Wolf, P.A.; Cobain, M.; Massaro, J.; Kannel, W.B. General Cardiovascular Risk Profile for Use in Primary Care: The Framingham Heart Study. Circulation 2008, 117, 743-753. [CrossRef]

19. Stern, M.P.; Williams, K.; Gonzalez-Villalpando, C.; Hunt, K.J.; Haffner, S.M. Does the Metabolic Syndrome Improve Identification of Individuals at Risk of Type 2 Diabetes and/or Cardiovascular Disease? Diabetes Care 2004, 27, 2676-2681. [CrossRef]

20. McNeill, A.M.; Rosamond, W.D.; Girman, C.J.; Golden, S.H.; Schmidt, M.I.; East, H.E.; Ballantyne, C.M.; Heiss, G. The Metabolic Syndrome and 11-Year Risk of Incident Cardiovascular Disease in the Atherosclerosis Risk in Communities Study. Diabetes Care 2005, 28, 385-390. [CrossRef]

21. Hu, G.; Qiao, Q.; Tuomilehto, J.; Balkau, B.; Borch-Johnsen, K.; Pyorala, K. Prevalence of the Metabolic Syndrome and Its Relation to All-Cause and Cardiovascular Mortality in Nondiabetic European Men and Women. Arch. Intern. Med. 2004, 164, 1066-1076. [CrossRef] [PubMed]

22. Wannamethee, S.G.; Shaper, A.G.; Lennon, L.T.; Morris, R. Metabolic Syndrome vs Framingham Risk Score for Prediction of Coronary Heart Disease, Stroke, and Type 2 Diabetes Mellitus. Arch. Intern. Med. 2005, 165, 2644-2650. [CrossRef] [PubMed]

23. Ormazabal, V.; Nair, S.; Elfeky, O.; Aguayo, C.; Salomon, C.; Zúñiga, F.A. Association between insulin resistance and the development of cardiovascular disease. Cardiovasc. Diabetol. 2018, 17, 122. [CrossRef] [PubMed]

24. Ragheb, R.; Shanab, G.M.; Medhat, A.M.; Seoudi, D.M.; Adeli, K.; Fantus, I. Free fatty acid-induced muscle insulin resistance and glucose uptake dysfunction: Evidence for PKC activation and oxidative stress-activated signaling pathways. Biochem. Biophys. Res. Commun. 2009, 389, 211-216. [CrossRef]

25. Roden, M.; Price, T.B.; Perseghin, G.; Petersen, K.F.; Rothman, D.L.; Cline, G.W.; Shulman, G.I. Mechanism of free fatty acid-induced insulin resistance in humans. J. Clin. Investig. 1996, 97, 2859-2865. [CrossRef]

26. Muniyappa, R.; Sowers, J.R. Role of insulin resistance in endothelial dysfunction. Rev. Endocr. Metab. Disord. 2013, 14, 5-12. [CrossRef] [PubMed]

(C) 2020 by the authors. Licensee MDPI, Basel, Switzerland. This article is an open access article distributed under the terms and conditions of the Creative Commons Attribution (CC BY) license (http://creativecommons.org/licenses/by/4.0/). 